\title{
Effet de la fertilisation azotée et phosphorée sur le développement de la Panachure jaune en riziculture aquatique en Côte d'Ivoire
}

\author{
Alphonse BOUET ${ }^{1 *}$, Nicaise Acho AMANCHO ${ }^{2}$, Souleymane SANOGO $^{3}$ et \\ Maméri CAMARA ${ }^{1}$ \\ ${ }^{1}$ Centre National de Recherche Agronomique (CNRA), BP 602 Gagnoa, Côte d'Ivoire. \\ ${ }^{2}$ Université d'Abobo-Adjamé, 02 BP 601 Abidjan 02, Côte d'Ivoire. \\ ${ }^{3}$ Université de Cocody-Abidjan UFR Biosciences, Laboratoire de Physiologie Végétale, 22 BP 582 Abidjan \\ 22, Côte d'Ivoire. \\ *Auteur correspondant, E-mail : bouetalph@yahoo.fr ; Tel : (225) 015010 93/(225) 07426485 , \\ Tél./Fax: (225) 32771700
}

\section{RESUME}

La crise mondiale du riz survenue en 2008 a montré l'impérative nécessité pour les pays tributaires des importations comme la Côte d'Ivoire, d'assurer leur autosuffisance. La fertilisation minérale figure parmi les cinq axes stratégiques définis pour l'accroissement de la production rizicole en Côte d'Ivoire. Sachant l'effet stimulateur de l'engrais azoté sur le développement de certaines maladies telle que la Pyriculariose du riz, un essai a été conduit dans le but de savoir l'influence d'une application optimale de l'azote sur l'épidémie de la Panachure jaune du riz ou Rice Yellow Mottle Virus (RYMV). L'étude a été réalisée selon un dispositif expérimental en SPLIT-PLOT comportant quatre niveaux d'azote $\left(0 \mathrm{~kg} \mathrm{~N} \mathrm{Ha}^{-1}, 30 \mathrm{~kg} \mathrm{~N} \mathrm{Ha}^{-1}, 60 \mathrm{~kg} \mathrm{~N} \mathrm{Ha}^{-1}\right.$, $\left.120 \mathrm{~kg} \mathrm{~N} \mathrm{Ha}^{-1}\right)$, trois niveaux de phosphore $\left(0 \mathrm{~kg} \mathrm{P} \mathrm{Ha}^{-1}, 13 \mathrm{~kg} \mathrm{P} \mathrm{Ha}^{-1}, 26 \mathrm{~kg} \mathrm{~N} \mathrm{Ha}^{-1}\right)$ et 10 génotypes de riz irrigué. Les résultats ont montré que le phosphore et l'interaction NxP n'ont aucun effet significatif sur l'épidémie de RYMV. A l'opposé, les analyses ont mis en évidence un effet significatif de l'azote sur l'incidence de la Panachure jaune à des doses comprises entre 0 et $30 \mathrm{Kg} \mathrm{N} \mathrm{Ha}^{-1}$. Cet intervalle de doses étant en deçà de celui des doses recommandées en Côte d'Ivoire, l'intensification de la riziculture aquatique par une fertilisation azotée optimale, n'aura pas d'incidence significative sur le développement de la Panachure jaune. Il faudra cependant sensibiliser les riziculteurs sur l'intérêt d'appliquer des doses d'engrais recommandées dans la lutte contre la virose.

(c) 2012 International Formulae Group. All rights reserved.

Mots clés: Riz irrigué, fertilisation minérale, épidémie, RYMV, incidence.

\section{INTRODUCTION}

La flambée des prix des denrées alimentaires de grande consommation telle que le riz, a provoqué en début de l'année 2008, un séisme social dans le monde, notamment dans les pays Afrique-CaraïbePacifique $(\mathrm{ACP})$. Face à cette fronde sociale devenue souvent incontrôlable, plusieurs gouvernements, suite aux mesures d'urgence palliatives décrétées, ont décidé d'assurer leur souveraineté alimentaire. Dans le cas de la Côte d'Ivoire qui importe (plus de $800000 \mathrm{~T}$ de riz blanchi en 2007) plus qu'elle ne produit (748000 T de riz blanchi en 2007) le riz, l'Etat a décidé d'accroître la production nationale de $140 \%$ à l'orée 2012, c'est-à-dire atteindre à cette échéance, $1500000 \mathrm{~T}$ de riz 
blanchi. Pour cela, la fertilisation organominérale a été préconisée parmi les moyens agronomiques à employer. Il est en outre envisagé de développer la riziculture aquatique (bas-fond, irriguée), naturellement plus productive que la riziculture pluviale.

Malheureusement, au cours de ces dix dernières années, une grande contrainte biotique est à la base de fortes pertes de récolte de riz dans l'écologie visée (aquatique). Il s'agit de la Panachure jaune ou Rice Yellow Mottle Virus (RYMV), une maladie virale causée par un sobemovirus. Décrite pour la première fois en 1970 au Kenya (Bakker, 1970), cette virose est aujourd'hui présente dans la plupart des zones de productions de riz en Afrique y compris le Madagascar (Abo et al., 1998; Traoré et al., 2001, Kouassi et al., 2005 ; Bouet et al., 2010). La maladie, circonscrite au continent africain, est transmise de façon mécanique par un complexe biotique et abiotique impliquant les insectes, des rongeurs et des ruminants puis aussi par le vent (Sarra and Peters, 2003; Sarra et al., 2004). Elle se manifeste par une décoloration des feuilles (panachure jaune), un retard de floraison, une sortie partielle puis une stérilité des épillets et un rabougrissement des plants infectés (Backer, 1974; Amancho et al., 2009). Les pertes de récolte dues au RYMV sont fréquentes et généralement importantes (29,5 à $100 \%$ en Côte d'Ivoire: Sanyang, 2006, Amancho et al., 2009; 56 à 100\% au Niger: Basso et al., 2006; 10 à $100 \%$ en Afrique: Awoderu, 1991, Kouassi et al., $2005 ; 19$ à 90,8\% en Sierra Leone : Fomba et al., 1986). En Côte d'Ivoire, la forte pression de la maladie a contraint dans plusieurs localités du pays, des paysans à l'abandon de leurs rizières (Vautrin, 1988).

Des études antérieures ont montré que la fertilisation minérale non rationalisée, notamment en azote, favorise le développement de certaines graves maladies $\mathrm{du}$ riz telle que la Pyriculariose ((Kozaka, 1965 ; Tokunaga et al., 1965, Bouet, 2008). Au niveau de la Panachure jaune du riz, très peu d'informations relatives à l'effet de la fertilisation minérale sur le développement de la maladie sont disponibles. Certains auteurs comme Henrichs et al. (2001) ont soupçonné l'azote de favoriser le développement de la
Panachure jaune (Henrichs et al., 2001). De plus, l'introduction des variétés exotiques et le changement des pratiques culturales en l'occurrence l'irrigation, l'emploi d'engrais et le maintien des cultures tout le long de l'année seraient à la base de la propagation du virus (Coulibaly et al., 2001). Une bonne connaissance du rôle des composantes de l'intensification dans l'épidémiologie du RYMV est donc nécessaire pour le succès des politiques de développement de la riziculture sous écosystème irrigué.

La présente étude a pour but de déterminer des doses optimales d'azote $(\mathrm{N})$ et de phosphore $(\mathrm{P})$ pour lesquelles la maladie de Panachure jaune, ne compromet pas les objectifs de production du riziculteur.

\section{MATERIEL ET METHODES}

Le matériel végétal était constitué de dix (10) génotypes de riz irrigué dont cinq (05) NERICAs, quatre (04) variétés Oryza sativa L. et un (01) cultivar traditionnel (Djoukemin), utilisé comme témoin local. Ce matériel a été soumis à la pression naturelle de la Panachure jaune (RYMV) sur la station expérimentale du Centre National de Recherche Agronomique (CNRA) de Gagnoa.

Le dispositif expérimental employé a été un SPLIT-PLOT avec, en parcelle principale, l'azote à 04 niveaux $(\mathrm{N} 0=0 \mathrm{~kg} \mathrm{~N}$ $\mathrm{Ha}^{-1} ; \mathrm{N} 1=30 \mathrm{~kg} \mathrm{~N} \mathrm{Ha}^{-1} ; \mathrm{N} 3=60 \mathrm{~kg} \mathrm{~N} \mathrm{Ha}^{-}$ ${ }^{1}$; $\left.\mathrm{N} 4=120 \mathrm{~kg} \mathrm{~N} \mathrm{Ha}^{-1}\right)$, en parcelle secondaire, 03 niveaux de $\mathrm{P}(\mathrm{P} 0=0 \mathrm{~kg} \mathrm{P} \mathrm{Ha}$ $\left.{ }^{1} ; \mathrm{P} 1=13 \mathrm{~kg} \mathrm{P} \mathrm{Ha}^{-1} ; \mathrm{P} 2=26 \mathrm{~kg} \mathrm{P} \mathrm{Ha}^{-1}\right)$ et en parcelle tertiaire, les génotypes de riz avec dix (10) modalités (de V1 à V10).

La parcelle élémentaire $(\mathrm{NxPxV})$ s'étendait sur $15 \mathrm{~m}^{2}$ (5 m x $3 \mathrm{~m}$ ) et comportait 375 plantes. Le semis a été réalisé par repiquage des plants issus d'une pépinière de 20 jours. Un seul apport de $\mathrm{P}$ a été fait au repiquage quand, l'N fut épandu au repiquage et au tallage.

Les mesures effectuées ont porté sur l'incidence, exprimée en pourcentage de plantes de riz attaquées par le RYMV à 70 JAR (jours après repiquage) et sur le rendement en paddy.

Les analyses statistiques (analyses de variance) ont été réalisées avec le logiciel GENSTAT au seuil de 5\%. Le test de 
comparaison des moyennes a été réalisé pour classer les différents traitements.

\section{RESULTATS}

Effet des facteurs étudiés (génotype de riz, azote et phosphore) sur l'incidence du RYMV

Les résultats de l'analyse de variance consignés dans le Tableau 1 montrent qu'aucune des interactions $(\mathrm{NxP}, \mathrm{NxG}, \mathrm{PxG}$, NxPxG) mises en évidence n'a eu d'effet significatif sur l'incidence de la Panachure jaune. Au niveau des facteurs pris individuellement, l'analyse révèle un effet significatif de l'azote $(\mathrm{N})$ et du génotype $(\mathrm{G})$ sur l'incidence du RYMV, contrairement au phosphore $(\mathrm{P})$.

\section{Réaction des génotypes de riz au RYMV}

Tous les génotypes de riz testés ont présenté des plants exhibant les symptômes caractéristiques de la Panachure jaune, avec des niveaux de sévérité identiques quel que soit le niveau de fertilisation. Cependant, des niveaux d'incidence variables ont été enregistrés en fonction des conditions de fertilisation. La pression de la maladie enregistrée sur les variétés est globalement faible. Elle varie de $1 \%$ à $7 \%$ et de $2,58 \%$ à $4,42 \%$ respectivement pour les parcelles non fertilisées et fertilisées. L'analyse statistique réalisée indique deux groupes variétaux (Tableau 2). Il s'agit, en l'absence de fertilisants, du groupe constitué par les variétés SATIVA FKR 64, WITA 4 et NERICA L 42. Les notes d'incidence affichées par ces variétés sont relativement les plus élevées, avec des valeurs moyennes supérieures ou égales à 3\%. Dans ce groupe de matériel sensible, SATIVA FKR 64 et WITA 4 captent l'attention avec des notes respectives de $7 \%$ et de $4 \%$. L'autre groupe comporte les génotypes ayant affiché des notes inférieures à $3 \%$. Dans ce dernier groupe, le cultivar traditionnel Djoukemin et la variété améliorée NERICA L 19 s'illustrent comme les plus résistants au RYMV.

En condition de fertilisation, encore deux groupes de réaction variétale s'identifient. Le premier groupe comprend les variétés WITA 4, SATIVA FKR 64, NERICA L20 NERICA L42 et NERICA L41, dont les notes d'incidence sont les plus élevées (comprises entre $3,70 \%$ et $4,50 \%$ ). Le dernier groupe comporte les autres variétés ayant affiché les plus faibles notes d'incidence (Tableau 2). Il est à noter par ailleurs que les variétés NERICA L41, NERICA L20, plus tôt résistantes en condition non fertilisée, sont parmi les plus sensibles en situation de fertilisation. En général, les notes d'incidence sont relativement plus élevées sous fertilisation qu'en l'absence d'engrais, sauf chez la variété SATIVA FKR 64 (Tableau 2).

Effet de l'azote et du phosphore sur l'incidence de la Panachure jaune

Sur la base des notes d'incidence enregistrées suite aux analyses de variance, sur chacun des génotypes de riz en condition avec ou sans fertilisation, deux constats s'imposent. Le premier est que $90 \%$ des génotypes de riz testés, présentent des valeurs d'incidence plus élevée dans les parcelles fertilisées que dans les parcelles non fertilisées. Le second est relatif au génotype SATIVA FKR 64 chez qui l'incidence du RYMV est plus élevée dans les parcelles non fertilisées que dans les parcelles fertilisées, contrairement aux autres (Tableau 2).

La courbe de la Figure 1 montre une augmentation de l'incidence de la maladie de la dose 0 à $30 \mathrm{~kg} \mathrm{~N} \mathrm{Ha}{ }^{-1}$, suivie d'une diminution jusqu'à la dose de $120 \mathrm{~kg} \mathrm{~N} \mathrm{Ha}^{-1}$ (Figure 1). En général, l'incidence de la panachure jaune est environ 1,5 fois plus élevée aux doses de $30 \mathrm{~kg} \mathrm{~N} \mathrm{Ha}^{-1}$ et $60 \mathrm{~kg} \mathrm{~N}$ $\mathrm{Ha}^{-1}$ qu'en situation sans engrais. L'épidémie semble régresser à partir des doses supérieures à $30 \mathrm{~kg} \mathrm{~N} \mathrm{Ha}{ }^{-1}$. Cependant, l'incidence de la maladie, dans l'ensemble, reste relativement plus élevée par rapport à la situation sans azote (Figure 1).

\section{Effet de la dose d'azote sur le rendement en paddy au champ}

Les niveaux de rendement en paddy varient avec la dose d'azote appliquée. Le rendement est passé de $3,43 \mathrm{~T} \mathrm{H}^{-1}$ à la dose de 0 kg N Ha ${ }^{-1}$ à 4,44 $\mathrm{T} \mathrm{Ha}^{-1}$ à la dose de $120 \mathrm{~kg}$ $\mathrm{Ha}^{-1}$. Le résultat de l'analyse statistique montre un effet significatif de la dose d'azote sur le rendement du riz. Les meilleurs rendements sont atteints aux doses de 60 et $120 \mathrm{~kg} \mathrm{~N} \mathrm{Ha}^{-1}$. En général, l'N a amélioré les rendements dans une proportion maximale de 
$29 \%$ par rapport au témoin sans engrais (Tableau 3). Par ailleurs, le rendement semble croître vers un optimum ou indéfiniment avec la dose d'azote.

\section{Niveau de production des génotypes de riz}

L'analyse de variance a mis en évidence l'effet significatif du génotype de riz sur le rendement en paddy. Aussi, deux types variétaux sont constitués. Le $1^{\mathrm{er}}$ groupe, avec $80 \%$ des variétés testées s'illustre par les rendements les plus élevés. Dans ce groupe, les variétés, SATIVA FKR 64, NERICA L 60, SATIVA BW 348-1 et WITA 4 ont présenté les meilleurs profils. Les rendements moyens chez ces variétés sont compris entre 4,272 T $\mathrm{Ha}^{-1}$ à 4,306 $\mathrm{Ta}^{-1}$ (Tableau 4). Il est à noter que les variétés SATIVA FKR 64 et WITA4 sont parmi les plus sensibles au RYMV (Tableau 2). Le $2^{\text {ième }}$ groupe comporte les variétés SATIVA FKR 19 et Djoukemin (Tableau 4), avec les plus faibles rendements. Remarquons que la variété traditionnelle Djoukemin, malgré son faible potentiel productif, est la plus résistante au RYMV (Tableau 2).

Tableau 1: Résultats de l'analyse de variance au seuil de 5\%.

\begin{tabular}{cccccc}
\hline Source de variation & DL & n & PPDS (lsd) & P & Effet \\
\hline Génotype de riz $(\mathrm{G})$ & 9 & 36 & 1,122 & 0,007 & $\mathrm{~s}$ \\
Azote $(\mathrm{N})$ & 3 & 90 & 0,958 & 0,037 & $\mathrm{~s}$ \\
Phosphore $(\mathrm{P})$ & 2 & 120 & 0,697 & 0,900 & $\mathrm{~ns}$ \\
NxP & 6 & 30 & 1,381 & 0,702 & $\mathrm{~ns}$ \\
NxG & 27 & 9 & 2,268 & 0,395 & $\mathrm{~ns}$ \\
PxG & 18 & 12 & 1,954 & 0,407 & $\mathrm{~ns}$ \\
NxPxG & 54 & 3 & 3,912 & 0,468 & $\mathrm{~ns}$ \\
\hline
\end{tabular}

DL = Degré de liberté ;PPDS (lsd = least significant difference of mean) = plus petite différence significative entre deux moyennes ; $\mathbf{P}=$ probabilité ; effet significatif si $\mathbf{P}<0,05 ; \mathrm{s}=$ significatif $; \mathrm{ns}=$ non significatif.

Tableau 2: Réactions de 10 génotypes de riz irrigué à la Panachure jaune (RYMV) en situation de fertilisation et de non fertilisation.

\begin{tabular}{lcc}
\hline Nom du génotype de riz & $\begin{array}{c}\text { Incidence du RYMV en } \\
\text { condition sans fertilisation }\end{array}$ & $\begin{array}{c}\text { Incidence du RYMV en } \\
\text { condition de fertilisation }\end{array}$ \\
\hline NERICA L19 & 1,33 & 2,58 \\
NERICA L20 & 2,33 & 3,81 \\
NERICA L41 & 2,33 & 3,75 \\
NERICA L42 & 3,00 & 3,80 \\
NERICA L60 & 1,67 & 3,03 \\
SATIVA FKR 19 & 2,33 & 2,86 \\
SATIVA FKR 64 & 7,00 & 4,17 \\
SATIVA BW 348-1 & 1,67 & 3,19 \\
WITA 4 & 4,00 & 4,42 \\
Djoukemin & 1,00 & 2,64 \\
Moyenne & 2,67 & 3,43 \\
Probabilité (P) & 0,030 & 0,007 \\
Effet & $\mathrm{s}$ & $\mathrm{s}$ \\
PPDS (lsd) & 3,104 & 1,122 \\
CV (\%) & 18,9 & 11 \\
\hline
\end{tabular}

$\mathrm{CV}=$ coefficient de variation $; \mathrm{ns}=$ non significatif $; \mathrm{s}=$ significatif $;$ effet significatif $\mathrm{si} \mathrm{P}<0,05$; deux moyennes sont différentes quant leur écart est supérieur au ppds ou lsd (plus petite différence significative). 
Tableau 3: Effet de la dose d'N sur le rendement en paddy.

\begin{tabular}{lc}
\hline Dose d'azote & Rendement en paddy $\left(\mathbf{T ~ H a}^{-1}\right)$ \\
\hline N0 $\left(0 \mathrm{kgNha}^{-1}\right)$ & 3,427 \\
$\mathrm{~N} 1\left(30 \mathrm{kgNha}^{-1}\right)$ & 3,960 \\
$\mathrm{~N} 2\left(60 \mathrm{kgNha}^{-1}\right)$ & 4,213 \\
$\mathrm{~N} 3\left(120 \mathrm{kgNha}^{-1}\right)$ & 4,443 \\
\hline Moyenne & 4,011 \\
Probabilité & 0,004 \\
Effet & $\mathrm{s}$ \\
PPDS $(1 \mathrm{sd})$ & 0,407 \\
CV $(\%)$ & 5,1 \\
\hline CV = Coefficient de Variation ; ns = non significatif ; s = significatif. Effet significatif si P $<0,05 ;$ deux \\
moyennes sont différentes quant leur écart est supérieur au ppds ou lsd (plus petite différence significatif).
\end{tabular}

Tableau 4: Valeurs moyennes de rendement en paddy $\left(\mathrm{T} \mathrm{Ha}^{-1}\right)$ obtenues sur les génotypes de riz étudiés.

\begin{tabular}{lc}
\hline Nom du génotype de riz & Rendement en paddy $\left(\mathbf{T ~ H a} \mathbf{~}^{-1}\right)$ \\
\hline NERICA L19 & 3,967 \\
NERICA L20 & 3,861 \\
NERICA L41 & 3,889 \\
NERICA L42 & 3,964 \\
NERICA L60 & 4,272 \\
SATIVA FKR19 & 3,794 \\
SATIVA FKR64 & 4,306 \\
SATIVA BW348-1 & 4,253 \\
WITA4 & 4,169 \\
DJOUKEMIN & 3,633 \\
\hline Moyenne & 4,011 \\
Probabilité & 0,001 \\
Effet & $\mathrm{s}$ \\
PPDS (lsd) & 0,225 \\
Cv (\%) & 12,100 \\
\hline CV = Coefficient de Variation ; ns = non significatif ; s = significatif. Effet significatif si P <0,05; \\
deux moyennes sont différentes quant leur écart est supérieur au ppds ou 1sd (plus petite différence \\
significatif).
\end{tabular}

\section{DISCUSSION}

La production locale de riz en Côte d'Ivoire ne satisfait qu'à peine $50 \%$ de la demande sociale de consommation. Pour résorber ce déficit de production estimé à plus de 800000 tonnes (en riz blanchi) et à terme parvenir à l'autosuffisance, l'Etat a défini cinq axes stratégiques. Au rang de ces axes, figure l'utilisation d'intrants agricoles, notamment l'engrais minéral. En riziculture aquatique (irrigué et de bas-fond) la dose d'engrais recommandée pour un rendement optimal est $200 \mathrm{~kg}$ de NPK (12 24 18) + $100 \mathrm{Kg}$ d'urée à l'hectare.

Cela représente en équivalent azote 70 $\mathrm{kg} \mathrm{N} \mathrm{Ha}{ }^{-1}$. Les résultats de la présente étude montrent qu'à une certaine dose, l'azote favorise le développement de la Panachure jaune, contrairement au phosphore et à l'interaction azote x phosphore qui n'ont eu aucun effet significatif sur l'épidémie du RYMV. 
A. BOUET et al. / Int. J. Biol. Chem. Sci. 6(6): 4071-4079, 2012

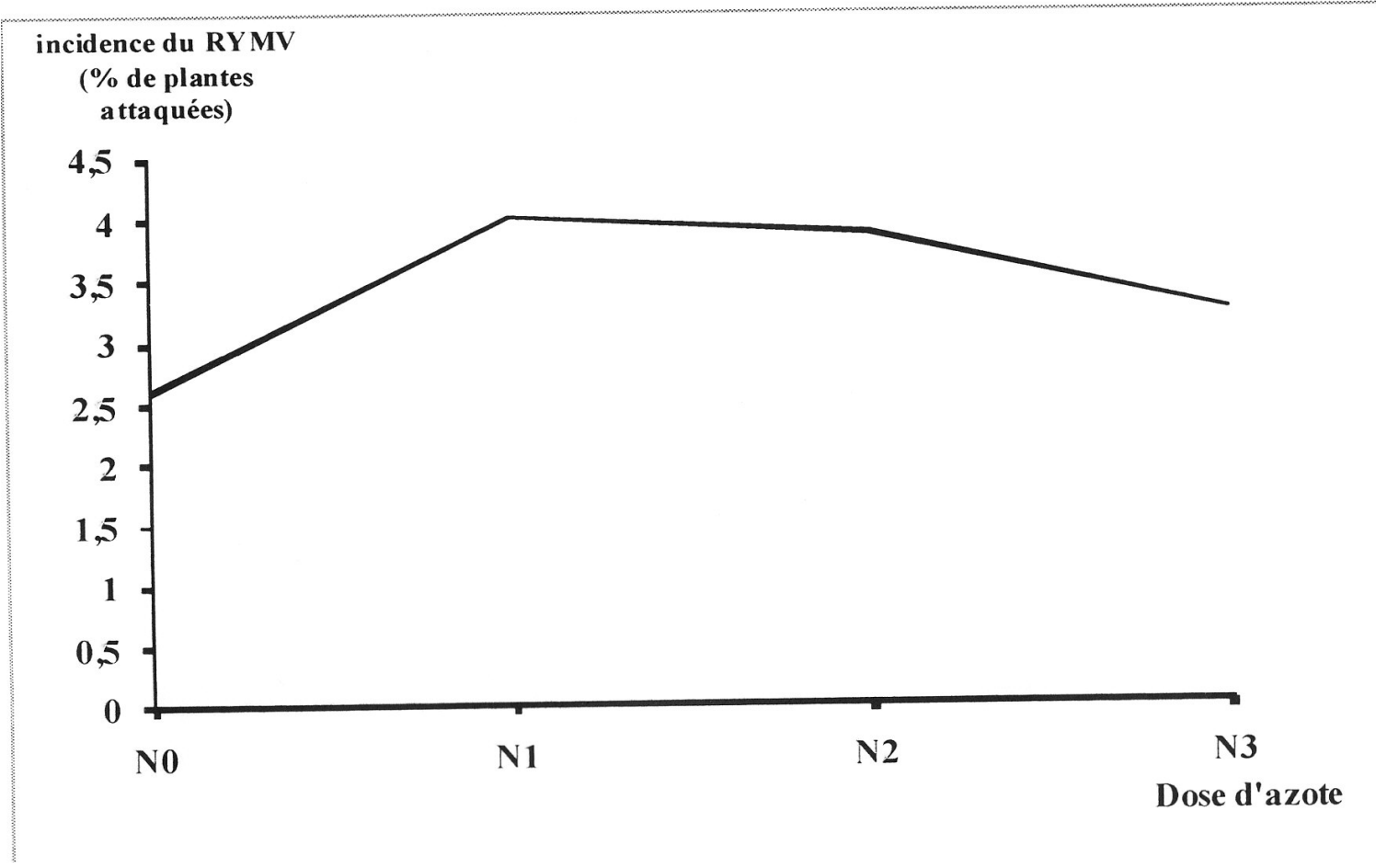

Figure 1 : Evolution de l'incidence du RYMV en fonction de la dose d'azote. 
Les riziculteurs du périmètre irrigué de l'office du Niger au Mali attribuaient déjà dans les années 1990, la forte pression du RYMV à l'application de l'urée. Ce constat a fait penser que l'intensification pourrait accentuer l'épidémie de la Panachure jaune (Coulibaly et al., 2001). Les travaux de Heinrichs et al. (2001) ont aussi révélé l'effet positif de l'N sur le taux d'infection du riz par le virus de la panachure jaune. L'information que fournit en plus la présente étude est que l'incidence du RYMV varie avec la dose d'N. Cette variation se traduit par une augmentation du pourcentage de talles infectées à des doses comprises entre 0 et 30 $\mathrm{kg} \mathrm{Ha}^{-1}$ et par la régression de la maladie à des doses supérieures à $30 \mathrm{~kg} \mathrm{Ha}^{-1}$. En sachant, d'après les travaux de Heinrichs et al. (2001) que la sévérité de la panachure est indifférente à l'azote, plusieurs raisons peuvent expliquer l'effet de l'N sur l'incidence de la Panachure jaune. L'une d'entre elles est que l'azote favorise à certaines doses, la création d'un microclimat propice à la ponte des insectes vecteurs du RYMV. Une autre est que l'azote empêche à certaines doses le développement des ennemis naturels des insectes vecteurs du RYMV.

Dans tous les cas et selon les résultats exposés, l'effet de l'N sur l'épidémie du RYMV n'est significatif qu'à des doses inférieures ou égales à $30 \mathrm{~kg} \mathrm{Ha}{ }^{-1}$. En comparant ces doses à celle recommandée en riziculture (200 kg Ha ${ }^{-1}$ NPK 101818 ou 12 $2418+100 \mathrm{~kg} \mathrm{Ha}^{-1}$ d'urée à $46 \% \mathrm{~N}$, soit 66 à $70 \mathrm{~kg} \mathrm{~N} \mathrm{Ha}^{-1}$ ), nous pouvons dire que l'intensification de la riziculture aquatique par la fertilisation ne sera pas un risque d'accentuation de l'épidémie du RYMVen Côte d'Ivoire. Il faut seulement craindre que pour des raisons financières, des paysans appliquent des doses d'N inappropriées, comprises entre 0 et $30 \mathrm{~kg} \mathrm{~N} \mathrm{Ha}{ }^{-1}$. Cette crainte est de mise d'autant que la plus part des riziculteurs dénoncent de manière récurrente, le coût élevé d'application de l'engrais. Au faible pouvoir financier des producteurs, une caractéristique générale de l'agriculture en Côte d'Ivoire, s'ajoute la précarité financière des structures d'encadrement et de développement, empêchant celles-ci d'assurer harmonieusement et régulièrement leurs missions d'appui-conseil. Face à ce constat d'actualité et pour éviter d'éventuels préjudices agricoles aux producteurs, il faut conseiller avant toute chose, la culture de variétés de riz résistantes ou tolérantes au RYMV. En effet, les risques de développement épidémique de la panachure jaune dus à la fertilisation azotée seront minimisés voire annulés avec ce type de matériel (résistant ou tolérant).

Par ailleurs, en se référant aux rendements obtenus sous les différentes doses d'azote, il ressort que les valeurs maximales sont obtenues avec les doses les plus élevées, comprises entre 60 et $120 \mathrm{~kg} \mathrm{~N} \mathrm{Ha}{ }^{-1}$. Cet intervalle de doses comportant la dose recommandée en riziculture aquatique en Côte d'Ivoire. En conséquence, la dose d'engrais minérale azotée, vulgarisée en riziculture irriguée, non seulement assure une bonne production mais aussi maintient la nuisance du RYMV à un niveau relativement faible.

L'effet stimulateur de l'azote sur l'épidémie des pathologies végétales a été déjà rapporté. Nous pouvons citer le cas de la Pyriculariose (Magnaporthe grisea, Herbert), une maladie fongique du riz, préoccupante en écologie pluviale. Il a été montré en effet que le développement de cette maladie est favorisé par des doses élevées d'azote (Kozaka, 1965 ; Tokunaga et al., 1965 ; Mohanty et Gangopadhyay, 1982 ; Bouet, 2008). Ces doses se situant à l'intérieur ou au-delà des recommandations. Dans le cas de la Panachure jaune, la pression épidémique est relativement forte à des doses d'azote inférieures à celles conseillées. Ainsi, les deux maladies sont sous l'influence de l'azote. Cependant, avec le RYMV, les objectifs de production ne sont pas compromis car une application optimale d'engrais peut être faite, contrairement à la Pyriculariose. En effet, en présence de la Pyriculariose, le souci de réduire ou de maintenir à un niveau faible l'épidémie, commande une application relativement réduite d'engrais.

L'autre information tirée de cette étude est que, dans un contexte épidémique préoccupant, le choix de la variété à cultiver ne doit pas se faire sur la base exclusive de 
son potentiel productif. Il faudra en plus considérer la réaction du matériel végétal à la maladie. Cette double exigence de la sélection permettra d'écarter des variétés telles que SATIVA FKR 64 et WITA4, très productives mais, parmi les plus sensibles à la Panachure jaune.

\section{Conclusion}

L'étude a montré que l'azote, appliqué à des doses comprises entre 0 et $30 \mathrm{~kg} \mathrm{~N}$ $\mathrm{Ha}^{-1}$ favorise le développement de la Panachure jaune. Cet intervalle de dose étant en deçà de celle des doses recommandées en Côte d'Ivoire, l'intensification de la riziculture aquatique par une fertilisation azotée normale, n'aura pas d'incidence significative sur le développement de la Panachure jaune. Il faudra cependant sensibiliser les riziculteurs sur l'intérêt d'appliquer des doses d'engrais recommandées dans la lutte contre la Panachure jaune.

\section{REMERCIEMENTS}

Nous remercions le centre de riz pour l'Afrique (Africa Rice) à la fois pour la mise à disposition des semences du matériel végétal étudié et l'appui financier apporté à la conduite des travaux.

\section{REFERENCES}

Abo M, Sy AA, Alegbejo M. 1998. Rice yellow mottle virus (RYMV) in Africa: Evolution distribution, economic significance and sustainable rice production and management strategies. Journal of Substanable Agriculture, 11: 85-111.

Amancho AN, Diallo AH, Kouassi KN, Bouet A, N'Guessan KP. 2009. Criblage de quelques variétés de riz de Côte d'Ivoire pour la résistance à la panachure du riz : incidence de la maladie sur quelques caractères agronomiques. Sciences et Natures, 6(1): 27-37.

Awoderu VA. 1991. The rice yellow mottle virus situation in West Africa. Journal of Basic Microbiology 31(2): 91-99.
Bakker W. 1970. Rice Yellow Mottle Virus a mechanically transmissible virus in Kenya. Neth J. Plant Pathol., 76: 53-63.

Bakker W. 1974. Characterization and ecological aspects of rice yellow mottle virus in Kenya. PhD Thesis, Agricultural University, Wageningen, the Netherlands, $152 \mathrm{pp}$.

Bassao A, Séré Y, Halidou A. 2006. Variabilité du virus de la panachure jaune du riz au Niger. In Proceedings of the 3rd regional rice research review (4Rs 2004), Narteh TL, Millard D, Beks B eds. 27 September- $1^{\text {st }}$ October 2004. Accra (Ghana): Africa Rice Center, pp. 145153.

Bouet A. 2008. Contribution à la lutte contre la pyriculariose, maladie fongique du riz : Oryza sativa L. (Poaceae) en Côte d'Ivoire: Caractéristiques de la population de l'agent pathogène : Magnaporthe grisea (Herbert) Barr (Ascomycetaceae) et méthodes d'évaluation variétale pour la résistance à la pyriculariose foliaire et à la pyriculariose du cou. Thèse de doctorat unique, Université de Cocody-Abidjan, $122 \mathrm{p}$

Coulibaly MY, Jamin YJ, Hamadou A, Ducrot R. 2001. La panachure jaune du riz (RYMV): Une menace pour l'intensification de la riziculture à l'office du Niger. In La Panachure Jaune du Riz. : Importance Economique, Diagnostic et Stratégie de Gestion, Sy AA, Hughes J, Diallo A eds. WARDA: M'bé, Bouaké, Côte d'Ivoire; 26-33.

Fomba SN. 1986. Crop losses and other effects of attack caused by the rice Yellow mottle virus (RYMV) on rice varieties, Rok5 and Angkata at Rokupr, Sierra Leone. West Africa Rice Development Association (WARDA). Technical Newsletter $\mathrm{N}^{\circ}$ 6.1, pp. 24-25.

Heinrichs AE, Sy AA, Akator KS, Oyediran I. 2001. Seasonal occurrence of rice Yellow Mottle Virus on the WARDA research farm. In Economic Importance, Diagnosis and Management, Sy AA, Hughes J, Diallo A (eds). WARDA: M'bé, Bouaké, Côte d'Ivoire; 59-63. 
Hebert TT. 1971. The perfect stage of Pyricularia grisea. Phytopathology, 61: 83-87.

Kouassi NK, N'Guessan P, Albar L, Fauquet CM, Brugidou C. 2005. Distribution and characterization of Rice yellow mottle virus: A threat to African Farmers. Plant disease, 2: 124-133.

Kozaka T. 1965. Control of rice blast by cultivation practices in Japan. In The Rice Blast Disease. Proceeding of a symposium at IRRI, IRRI (ed). Johns Hopkins Press: Baltimore, Maryland, USA; 421-438.

Mohanty CR, Gangopadhyay S. 1982. Testing of blast resistance in $\mathrm{F} 2$ rice seedlings in different doses of nitrogen and season. Ann. Phytopath. Soc. Japan, 48: 648-658.

Sanyang S. 2006. $3^{\text {rd }}$ biennial regional rice research review (4Rs 2004). A statut report of ROCARIZ. 27 September $-1^{\text {st }}$ October 2004, Accra (Ghana): Africa Rice Center.
Sarra S, Peters D. 2003. Rice yellow mottle virus is transmitted by cows, donkeys, and grass rats in irrigated rice crops. Plant Disease, 87: 804-808.

Sarra S, Oevering P, Guindo S, Peters D. 2004.Wind-mediated spread of Rice yellow mottle virus (RYMV) in irrigated rice crop. Plant Pathology, 53: 148-153.

Tokunaga Y, Kato H, Koshimiza Y. 1965. Studies on the relationship between metabolism of rice and its resistance to blast disease; 2 effect of the phosphorus content in leaf blades during maturity on disease proneness to leaf blast. Bull. Tohoku natl. Agric. Exp. Station, 32: 6187.

Traore' O, Pinel A, Fargette D, Konate' G. 2001. First report and characterization of Rice yellow mottle virus in Central Africa. Plant Disease, 85: 92.

Vautrin. 1988. Requête d'intervention sur une maladie dans les rizières de la région du Projet PACO. 